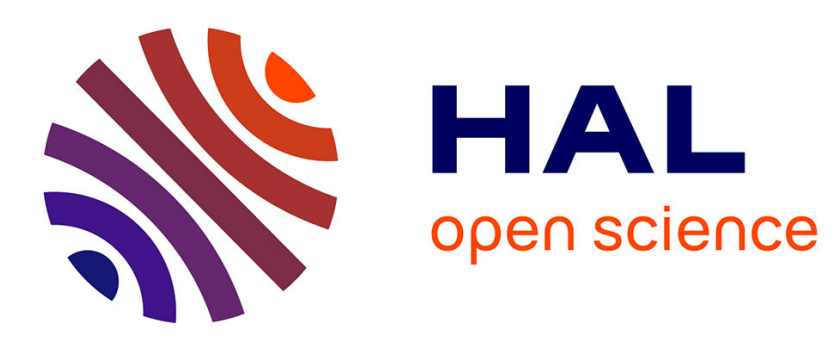

\title{
Definition, properties and wavelet analysis of multiscale fractional Brownian motion
}

Jean-Marc Bardet, Pierre, Raphael Bertrand

\section{To cite this version:}

Jean-Marc Bardet, Pierre, Raphael Bertrand. Definition, properties and wavelet analysis of multiscale fractional Brownian motion. Fractals, 2007, 15 (1), pp.73-87. hal-00127938

\section{HAL Id: hal-00127938 \\ https://hal.science/hal-00127938}

Submitted on 30 Jan 2007

HAL is a multi-disciplinary open access archive for the deposit and dissemination of scientific research documents, whether they are published or not. The documents may come from teaching and research institutions in France or abroad, or from public or private research centers.
L'archive ouverte pluridisciplinaire HAL, est destinée au dépôt et à la diffusion de documents scientifiques de niveau recherche, publiés ou non, émanant des établissements d'enseignement et de recherche français ou étrangers, des laboratoires publics ou privés. 


\title{
Definition, properties and wavelet analysis of multiscale fractional Brownian motion
}

\author{
September 21, 2006
}

\author{
JEAN-MARC BARDET* and PIERRE BERTRAND** \\ * CES-MATISSE-SAMOS - UMR CNRS 8174, Université Panthéon-Sorbonne (Paris I), 90 rue \\ de Tolbiac, 75013 Paris Cedex, France, E-mail: bardet@univ-paris1.fr \\ ** Laboratoire de Mathématiques - UMR CNRS 6620, Université Blaise Pascal (Clermont-Ferrand \\ II), 24 Avenue des Landais, 63117 Aubière Cedex, France. E-mail: Pierre.Bertrand@math.univ- \\ bpclermont.fr
}

\begin{abstract}
In some applications, for instance finance, biomechanics, turbulence or internet traffic, it is relevant to model data with a generalization of a fractional Brownian motion for which the Hurst parameter $H$ is depending on the frequency. In this contribution, we describe the multiscale fractional Brownian motions which present a parameter $H$ as a piecewise constant function of the frequency. We provide the main properties of these processes: long-memory and smoothness of the paths. Then we propose a statistical method based on wavelet analysis to estimate the different parameters and prove a functional Central Limit Theorem satisfied by the empirical variance of the wavelet coefficients.
\end{abstract}

Keywords: Fractional Brownian motion; Long-range dependence; Path regularity; Self-similarity; Wavelet analysis; functional Central Limit Theorem.

\section{Introduction}

Fractional Brownian Motion (F.B.M.) was introduced in 1940 by Kolmogorov as a way to generate Gaussian "spirals" in a Hilbert space. But the seminal paper of Mandelbrot and Van Ness (1968) emphasizes the relevance of F.B.M. to the modelling of natural phenomena: hydrology, finance,... Various properties of F.B.M. such as self-similarity and smoothness of the sample paths, long range dependence of its increments, are related to the Hurst parameter H. During the decades 1970's and 1980's, the statistical study of F.B.M. has been developed, see for instance the historical notes in 
Samorodnitsky \& Taqqu (1994), [29, chap.14] and the references therein. F.B.M. has been more and more used in several areas during the last decade (internet traffic, turbulence, image processing...). Anyway, in many applications the real data do not fit exactly F.B.M. which appears as an ideal mathematical model. Therefore different generalizations of F.B.M. have been proposed these last years to fill the gap between the mathematical modelling and real data.

One example is the family of model derived from the Multifractional Brownian Motion (M.B.M.) introduced independently in Peltier and Lévy Vehel (1996) and Benassi, Jaffard and Roux (1997). For the M.B.M. the Hurst parameter $H$ is replaced by a function depending continuously on the time $t \mapsto H(t)$. Afterwards, the Generalized Multifractional Brownian Motion (G.M.B.M.) has been introduced by Ayache and Lévy Vehel (2000) for modelling the situations where the Hurst parameter $H$ should be replaced by an irregular function of time. Nevertheless, in some fields (image analysis or control of internet traffic) the interesting information is the location of changes point of the function $t \mapsto H(t)$ and, in these cases, $H($.$) appears as a piecewise constant function$ of time. Benassi et al. (2000) proposed the Step Fractional Brownian Motion (S.F.B.M.) as a good model in this circumstance. The detection of changes times of the function $t \mapsto H(t)$ for the S.F.B.M. has been investigated in Benassi et al. (2000) and Ayache, Bertrand and Lévy Vehel (2006).

So finding a good generalization of the F.B.M. enhancing the goodness of fit to the different applications has became an active field of research. A first stream which has been described above, is concerned with Gaussian processes where the Hurst parameter $H$ has been replaced by a function depending on the time. This dependency of time induces the loss of the stationarity of the increments. A second stream is concerned with non Gaussian processes, mainly $\alpha$ stable $(0<\alpha<2)$ infinite variance processes, see for example the study of telecom processes in Pipiras and Taqqu (2002).

In this work, we are concerned with Gaussian processes with stationary increments where the Hurst parameter $H$ is replaced by a piecewise constant function of the frequency $\xi \mapsto H(\xi)$. Before going further, we recall a precise definition of the F.B.M. and some of its properties.

Formally, a fractional Brownian motion $B_{H}=\left(B_{H}(t), t \in \mathbb{R}_{+}\right)$could be defined as a real centered Gaussian process with stationary increments such that $B_{H}(0)=0$ and $\mathbb{E}\left|B_{H}(s)-B_{H}(t)\right|^{2}=$ $\sigma^{2}|t-s|^{2 H}$, for all pair $(s, t) \in \mathbb{R}_{+} \times \mathbb{R}_{+}$where $\left.H \in\right] 0,1[$ and $\sigma>0$. This process is characterized by two parameters: the Hurst index $H$ and the scale parameter $\sigma$. We lay the emphasis on the fact the same parameter $H$ is linked to three different properties of the F.B.M.:

1. The self-similarity of the process, i.e. for all $\lambda \in \mathbb{R}^{+}$,

$$
\left(B_{H}(\lambda t)\right)_{t \in \mathbb{R}_{+}} \stackrel{(d)}{=}\left(\lambda^{H} B_{H}(t)\right)_{t \in \mathbb{R}_{+}} .
$$

2. The regularity of the sample paths: with probability one, the map $t \mapsto B_{H}(t)$ is Hölder continuous of order $\alpha$, for all positive real number $\alpha<H$;

3. The long memory (or short memory) property of the increments. Let $Y(t)=B_{H}(t+1)-B_{H}(t)$, for $t \in \mathbb{R}_{+}, Y$ is a centered stationary Gaussian process corresponding to the increment of the 
process $B_{H}$. A stationary $\mathbb{L}^{2}$-process $Y=(Y(t), t \in \mathbb{N})$ is said to be a long-range dependent process (or long-memory process) when

$$
\sum_{k=-\infty}^{\infty}|r(k)|=\infty
$$

where $r(k)=\operatorname{cov}(Y(k), Y(0))$ is the covariogram of $Y$. For the F.B.M. we have $|r(k)| \sim$ $\sigma^{2} H(2 H-1) k^{2 H-2}$ when $|k| \rightarrow+\infty$ (and $H \neq 1 / 2$, the case of $H=1 / 2$ corresponding to the well-known case of Brownian motion with independent increments). Thus, when $H>1 / 2$, the increments of a F.B.M. are long-memory process and when $H \leq 1 / 2$, a short-memory process.

From one hand, the regularity of the sample paths could be interpreted as a high frequency behavior, from the other hand the long memory of the increments correspond to a low frequency behavior. Yet, as mentioned by Mandelbrot and Van Ness (1968) [24], "the concept of self-similarity is a form of invariance with respect to changes of time scale ". For this reason, the high frequency behavior and the low frequency behavior are linked and driven by the same parameter $H$. However, in some applications, this link can appear as artificial. We indicate below three examples in finance, turbulence and biomechanics

- On the one hand, statistical studies on financial data show the long memory property of the price process, see for e.g. Willinger et al. [30]. On the other hand, financial theory says it should not be possible to make profit without any risk. This implies that the price process should be a semi-martingale, therefore its Hurst index should be $H=1 / 2$ at high frequencies. In this case the contradiction between the empirical evidence and the theoretical reasoning follows from the invariance with respect to changes of time scale. This contradiction would disappear if one put an end to this scale-invariance assumption.

- In turbulence phenomena, the assumption of scale-invariance is assumed within the inertial range (see Frisch [22, p.104] or Papanicolaou and Sølna (2002) [25]). Fundamental and experimental physics show that the scale invariance is valid for a large but finite range of scales excluding both the microscopically and the astronomically ones.

- In biomechanics, Collins and de Luca (1993) introduced a fractional process with two different Hurst indexes at low and high frequencies, for modelling the trajectories of the center of pressure of human been in upright position. Moreover, in this case the relevant information is the value of the change frequency.

The previous examples exhibit the advantage to model real data by Gaussian processes having stationary increments with a Hurst index varying as a piecewise function of the frequencies. To our knowledge, these kinds of processes have been introduced implicitly in biomechanics by Collins and de Luca (1993), in finance by Rogers (1997) and Cheridito (2003) and explicitly by Benassi and Deguy (1999) for image analysis and image synthesis. Anyway, the probabilistic properties of these processes have never been precisely established and no rigorous statistical study has been 
made. Both Collins and de Luca (1993) and Benassi and Deguy (1999) propose a model with two different Hurst indices corresponding respectively to the high and the low frequencies separated by one change point at the frequency $\omega_{c}$. They use the log-variogram to estimate these two Hurst indices. Indeed, in this case, the log-variogram considered as a function of the logarithm of the scale presents two asymptotic direction with slopes being twice the Hurst index at low (respectively high) frequencies. The change point $\omega_{c}$ is then estimated as the X-coordinate of the intersection of these two straight lines. Numerically, this method is not robust. Moreover it could not be adapted in the case of more than one change point. Let us stress this is not a theoretical refinement, but corresponds precisely to the real situations. Indeed, in applications, we only consider finite bands of frequencies, therefore we should use statistical method based on the information included in finite bands of frequencies. Wavelet analysis appears as the had hoc tool, when the Fourier transform of the associated wavelet is compactly supported.

For these reasons, we propose a model including the cases with more than one frequency change point and investigate the estimation of the Hurst function $\xi \mapsto H(\xi)$ inside finite bands of frequencies by the mean of wavelet analysis. The remainder of the paper is organized as follows: in section 2, we define the multiscale fractional Brownian motion and establish its main properties: the long memory one and the smoothness of sample paths. In section 3, we derive a statistical study based on its wavelet analysis. More precisely, the empirical variance of wavelet coefficients provides a semi-parametric method for estimating the different parameters of the process. For this purpose, we prove a functional Central Limit Theorem satisfied by the empirical variance of the wavelet coefficient considered as a function of the scale. In the appendices A1 and A2, we develop two examples of applications in finance and biomechanics. The proofs of section 2 and 3 are respectively given in the appendices $\mathrm{B} 1$ and $\mathrm{B} 2$.

\section{Description of the Model and its Probabilistic Properties}

In this section, the multiscale fractional Brownian motion is defined and its probabilistic properties, mainly the smoothness of the paths and the long-memory property, are studied.

\subsection{Definition of the Multiscale Fractional Brownian Motion}

Fractional Brownian motions (F.B.M.) were popularized by Mandelbrot and Van Ness (1968) who suggest the study of their properties as a typical example of non-Markovian process. F.B.M. are Gaussian processes with stationary increments with a given covariance structure. The F.B.M. has different representations which could alternately be used as definition: the moving average representation, the harmonizable representation. We propose Samorodnitsky and Taqqu (1994), [29, chap. 7] as a reference book on the matter. The harmonizable representation of the F.B.M. $([29$, , p. 328$]$ is well adapted to our problem and is defined by

$$
B_{H}(t)=\int_{\mathbb{R}} \frac{\left(e^{i t \xi}-1\right)}{|\xi|^{H+1 / 2}} \widehat{W}(d \xi)
$$


where $W(d x)$ is a Brownian measure and $\widehat{W}(d \xi)$ its Fourier transform, namely for any function $f \in L^{2}(\mathbb{R})$ one has almost surely, $\int_{\mathbb{R}} f(x) W(d x)=\int_{\mathbb{R}} \widehat{f}(\xi) \widehat{W}(d \xi)$, with the convention that $\widehat{f}(\xi)=\int_{\mathbb{R}} e^{-i \xi x} f(x) d x$ when $f \in L^{1}(\mathbb{R}) \cap L^{2}(\mathbb{R})$.

A natural generalization consists in replacing the Hurst index $H$ in formula (1) by a piecewise function depending on frequencies, i.e. $\xi \mapsto H(\xi)$. We call it the multiscale fractional Brownian motion and we define it as follows,

Definition 2.1 For $K \in \mathbb{N}$, a $\left(M_{K}\right)$-multiscale fractional Brownian motion (simplify by $\left(M_{K}\right)$ F.B.M.) $X=\left(X(t), t \in \mathbb{R}_{+}\right)$is a process such that

$$
X(t)=\int_{\mathbb{R}} \frac{\left(e^{i t \xi}-1\right)}{\rho(\xi)} \widehat{W}(d \xi) \text { for } t \in \mathbb{R}_{+}
$$

with

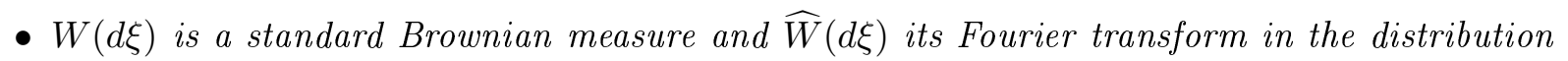
meaning.

- for $i=0, \ldots, K$, there exist $\left(\omega_{i}, \sigma_{i}, H_{i}\right) \in\left(\mathbb{R}_{+}, \mathbb{R}_{+},\right] 0,1[)$ such that $\rho(\xi)=\sigma_{i}^{-1}|\xi|^{H_{i}+1 / 2}$ for $|\xi| \in\left[\omega_{i}, \omega_{i+1}\left[\right.\right.$ with $\omega_{0}=0<\omega_{1}<\ldots<\omega_{K}<\omega_{K+1}=+\infty$ by convention.

The $\left(M_{K}\right)$-F.B.M. was first introduced in Benassi and Deguy (1999) in the case of only one change. A particular case of such a process can also be found in Ayache and Lévy-Vehel (2004). Until the end of this article, we restrict ourselves to one dimensional processes depending on a parameter $t \in \mathbb{R}_{+}$, but a generalization to random fields could also be studied.

Remark 2.1 Formula (2) defined a zero-mean Gaussian process as soon as

$$
\mathcal{J}=\int_{\mathbb{R}}\left|e^{i t \xi}-1\right|^{2} \rho^{-2}(\xi) d \xi<\infty .
$$

But $\mathcal{J}=2 \sum_{i=0}^{K} \int_{\omega_{i}}^{\omega_{i+1}}[1-\cos (t \xi)] \times \xi^{-\left(2 H_{i}+1\right)} d \xi$, therefore the conditions $H_{K}>0$ and $H_{0}<1$ insure respectively the convergence at point $\infty$ and at point 0 and after imply $\mathcal{J}<\infty$. To sum up, for all $\left(H_{0}, \ldots, H_{K}\right) \in \mathbb{R}^{K+1}$ with $H_{K}>0$ and $H_{0}<1$, formula (2) defined a zero-mean Gaussian process. Moreover, when $H_{K}<1$ Property 2.1, item 2 and item 3, i.e. formulae (3) and (4) below, remain in force. For this new generalization, the difference concerns the long-memory property of the increments. Indeed, the properties (5) and (6) below are also satisfied but the proof has to be changed when $H_{0} \leq 0$ (it is straightforward...).

\subsection{Some Probabilistic Properties of the Multiscale Fractional Brownian Mo- tion}

The proof of the existence of a continuous version of a $\left(M_{K}\right)$-F.B.M. can be found in Cramér and Leadbetter (1967). A $\left(M_{K}\right)$-F.B.M. presents $K$ spectral change points. A $\left(M_{0}\right)$-F.B.M. is a fractional Brownian motion with a Hurst parameter $H_{0}$. A $\left(M_{K}\right)$-F.B.M. has the same expression than a fractional Brownian motion with a Hurst parameter $H_{i}$ for frequencies $|\xi| \in\left[\omega_{i}, \omega_{i+1}[\right.$. 
Therefore we could expect that a $\left(M_{K}\right)$-F.B.M. path would have the regularity driven by $H_{K}$ (at high frequencies) and a long term dependence of its increments driven by $H_{0}$ (at low frequencies). The first point is satisfied, but Property 2.2 shows that the second one is a little bit more complicated.

Property 2.1 Let $X$ be a $\left(M_{K}\right)$-F.B.M.

1. $X$ is a Gaussian centered process with stationary increments.

2. For a time step $\delta>0$, let the variogram of $X$ be $\mathcal{V}(\delta):=\mathbb{E}(X(t+\delta)-X(t))^{2}$. Then we have

$$
\mathcal{V}(\delta)=4 \sum_{i=0}^{K} \delta^{2 H_{i}} \sigma_{i}^{2} \int_{\delta \omega_{i}}^{\delta \omega_{i+1}} \frac{(1-\cos v)}{v^{2 H_{i}+1}} d v .
$$

3. We have the decomposition

$$
X(t)=B_{H_{K}}(t)+R(t)
$$

where $B_{H_{K}}$ is a F.B.M. with Hurst index $H_{K}$ and $R$ a continuous process with finite variation. Thus $X$ has the same regularity than the F.B.M. $B_{H_{K}}$. Particularly, the trajectories of $X$ are a.s. of Hölder regularity $\alpha$, for every $\alpha \in\left[0, H_{K}[\right.$.

Therefore a $\left(M_{K}\right)$-F.B.M. shares two properties with a F.B.M. of parameter $H_{K}$ : they are continuous Gaussian centered processes with stationary increments and $\alpha$-Hölderian regularity for every $\alpha \in\left[0, H_{K}[\right.$. However, Formula (3) shows a substantial difference between both the processes: for all $\delta \in \mathbb{R}_{+}$, the variance of a $\left(M_{K}\right)$-F.B.M. in $\delta$ is a function depending on $\delta$ and all the parameters $\left(H_{i}, \omega_{i}, \sigma_{i}\right)_{0 \leq i \leq K}$. Thus, the variance of a $\left(M_{K}\right)$-F.B.M. is not a self-similar function and the famous log-variogram method is not relevant for the estimation of the different parameters.

Now, in view of the study of the low frequency behavior, we consider process of the increments of $X, Y=\left(Y(t), t \in \mathbb{R}_{+}\right)$, with

$$
Y(t)=X(t+1)-X(t), \quad \text { for } t \in \mathbb{R}_{+} .
$$

Remark 2.2 It is obvious that for all $\delta>0$, the process $Y_{\delta}$ such that $Y_{\delta}=\left(Y_{\delta}(t), t \in \mathbb{R}_{+}\right)=$ $\left(X(t+\delta)-X(t), t \in \mathbb{R}_{+}\right)$is also a stationary Gaussian process. The following property 2.2 can also be established for $\left(Y_{\delta}(t)\right)_{t}$ instead of $(Y(t))_{t}$. The only difference is that each $\sigma_{i}^{2}$ has to be multiplied by a factor $\delta^{2 H_{i}}$ in the right term of formula (5) below and also the different terms of the following polynomial $P(n)$ in formula (7).

$Y$ is a centered stationary Gaussian process. Moreover, we have the following property:

Property 2.2 Let $X$ be a $\left(M_{K}\right)$-F.B.M. with $K \geq 1$ and $Y$ its increments defined as previously. Define $r(n)=\operatorname{cov}(Y(n), Y(0))$, the correlogram of the increments of $X$. Then

$$
\begin{aligned}
& \text { if } H_{0}>\frac{1}{2}, \quad r(n)=\left(\frac{\pi \sigma_{0}^{2}\left(2 H_{0}-1\right)}{\Gamma\left(2 H_{0}\right) \sin \left(\pi H_{0}\right)}\right) \frac{1}{n^{2-2 H_{0}}}+\mathcal{O}\left(\frac{1}{n}\right), \\
& \text { if } H_{0} \leq \frac{1}{2}, \quad r(n)=\frac{P(n)}{n}+\mathcal{O}\left(\frac{1}{n^{2-2 H_{0}}}\right),
\end{aligned}
$$


with $P(n)$ a trigonometric polynomial depending only on $\left(H_{i}, \omega_{i}, \sigma_{i}\right)_{0 \leq i \leq K}$ such that

$$
P(n)=8 \sum_{i=1}^{K} \sin ^{2}\left(\frac{\omega_{i}}{2}\right) \times\left(\frac{\sigma_{i-1}^{2}}{\omega_{i-1}^{2 H_{i}-1}}-\frac{\sigma_{i}^{2}}{\omega_{i}^{2 H_{i}}}\right) \times \sin \left(n \omega_{i}\right) .
$$

From this property we derive the asymptotic behavior of the dependence of the increments of $X$. Then, the long-range property of the increments of the process $Y$ depends on $H_{0}$, but not only on this value. More precisely, if $H_{0}>1 / 2$, the long-range (or low frequencies) behavior of $Y$ is the same that the behavior of a fractional Gaussian noise with parameter $H_{0}$. Thus, $Y$ is a long-range dependent process. If $H_{0} \leq 1 / 2$, the process $Y$ is a long-range dependent process except when $\left(H_{i}, \omega_{i}, \sigma_{i}\right)_{0 \leq i \leq K}$ satisfies a relation such that the trigonometric polynomial $P(n)$ of $(7)$ is vanished.

Remark 2.3 If we consider the spectral density function of the stationary process $Y$ in the meaning of Cramér and Leadbetter (1967, p. 136), i.e. the function $f$ such that $r(t)=\int_{0}^{\infty} \cos (\lambda t) f(t) d t$, we have $f(t)=\frac{\sin ^{2}(t / 2)}{\rho^{2}(t)}$. Therefore, $f$ is not a continuous function except if for all $i=1, \cdots, K$, $f\left(\omega_{i}\right)=\lim _{t \rightarrow \omega_{i}^{-}} f(t)$, i.e. the same condition that $P(n)=0$ for all $n \in \mathbb{N}$. Since $f$ is not a continuous function then $\sum_{k \in \mathbb{N}}|r(k)|=\infty$ and thus $Y$ is a long-range dependent process. For instance, if $K=1$ and $\frac{\sigma_{0}^{2}}{\omega_{1}^{2 H_{0}+1}} \neq \frac{\sigma_{1}^{2}}{\omega_{1}^{2 H_{1}+1}}, f$ is discontinuous in $\omega_{1}$ and $Y$ is a long-range dependent process even if $H_{0}<1 / 2$ and $H_{1}<1 / 2$.

\section{A statistical study based on wavelet analysis}

In this section, we propose a statistical study based on wavelet analysis. After having explained the reason of this choice, we describe the definition of the wavelet coefficients and their basic properties. Then we state the main result of this section, that is a functional central limit theorem satisfied by the empirical variance of wavelet coefficients. At the end of this section, we give a numerical example which illustrate the Central Limit Theorem.

To begin with, we specify the statistical framework. Let $X$ be a $\left(M_{K}\right)$-F.B.M. defined by (2). We observe one path of the process $X$ on the interval $\left[0, T_{N}\right]$ at the discrete times $t_{i}=i \times \Delta_{N}$ for $i=1, \ldots, N$ and $T_{N}=N \times \Delta_{N}$. We want to estimate the parameters of the $\left(M_{K}\right)$-F.B.M. that is $\left(H_{0}, H_{1}, \ldots, H_{K}\right),\left(\sigma_{0}, \sigma_{1}, \ldots, \sigma_{K}\right)$ and $\left(\omega_{1}, \ldots, \omega_{K}\right)$. We consider the asymptotic $N \rightarrow+\infty$, $\Delta_{N} \rightarrow 0$ and $T_{N} \rightarrow+\infty$.

Even if the model is defined here as a parametric one, we prefer to use a semi-parametric statistics for different reasons. First, the covariance of the increments has the asymptotic behavior described in Property 2.2 and if $H_{0}<1 / 2$, this behavior cannot be written as $r(n)=L(n) n^{-d}$ with $L$ a slow varying function. Then, the classical results (see Fox and Taqqu, 1986, Dahlhaus, 1989 or Giraitis and Surgailis, 1990) on the asymptotic behaviors of parametric (maximum likelihood estimator and Whittle maximum likelihood) estimators cannot be used. As a consequence, a new and difficult study of the convergence rate of such estimators should be done, without any guarantee to obtain 
good properties (another difficulty is implied by the discontinuity of the spectral density function as seen in Remark 2.3).

Afterwards, the following semi-parametric statistics is more robust than a parametric one if the model is misspecified. Consider the example where the function $H(\xi)$ is a not exactly a piecewise constant function, but instead a constant function on several intervals (in other intervals, $H(\xi)$ is some unknown function). In this case, a parametric estimator could not work while the following semi-parametric method will remain relevant.

Eventually, we prefer to use a method based on a wavelet analysis. This method has been introduced by Flandrin (1992) and was developed by Abry et al. (2001) and Bardet et al. (2000). Let $\psi$ be a "mother" wavelet satisfying the following assumption:

Assumption (A1): $\psi: \mathbb{R} \mapsto \mathbb{R}$ is a $\mathcal{C}^{\infty}$ function satisfying:

- for all $m \in \mathbf{Z}, \int_{\mathbb{R}}\left|t^{m} \psi(t)\right| d t<\infty$,

- its Fourier transform $\widehat{\psi}(\xi)$ is an even function compactly supported on $[-\beta,-\alpha] \cup[\alpha, \beta]$ with $0<\alpha<\beta$.

We stress these conditions are sufficiently mild and are satisfied in particular by Lemarié-Meyer wavelet. The admissibility property, i.e. $\int_{\mathbb{R}} \psi(t) d t=0$ is a consequence of the second one and more generally, for all $m \in \mathbb{N}$,

$$
\int_{\mathbb{R}} t^{m} \psi(t) d t=0
$$

Remark that it is not necessary to choose $\psi$ being a "mother" wavelet associated to a multiresolution analysis of $\mathbb{L}^{2}(\mathbb{R})$. The whole theory can be developed without resorting to this assumption: the choice of $\psi$ is then very large.

Let $(a, b) \in \mathbb{R}_{+}^{*} \times \mathbb{R}$, we denote $\lambda=(a, b)$. Then we define the family of functions $\psi_{\lambda}$ by $\psi_{\lambda}(t)=\frac{1}{\sqrt{a}} \psi\left(\frac{t}{a}-b\right)$. Parameters $a$ and $b$ are so-called the scale and the shift of the wavelet transform (here we consider a continue wavelet transform). Let $d_{X}(a, b)$ be the wavelet coefficient of the process $X$ for the scale $a$ and the shift $b$, with

$$
d_{X}(a, b)=\frac{1}{\sqrt{a}} \int_{\mathbb{R}} \psi\left(\frac{t}{a}-b\right) X(t) d t=<\psi_{\lambda}, X>_{L^{2}(\mathbb{R})} .
$$

This family of wavelet coefficients satisfies the following properties:

Property 3.1 Let $\psi$ satisfy Assumption (A1) and $X$ be a $\left(M_{K}\right)$-F.B.M. Denote

$$
\mathcal{I}_{1}(a)=a \int_{\mathbb{R}}|\widehat{\psi}(a u)|^{2} \rho^{-2}(u) d u .
$$

Then:

1. for $a>0,\left(d_{X}(a, b)\right)_{b \in \mathbb{R}}$ is a stationary centered Gaussian process such that:

$$
d_{X}(a, b)=\int_{\mathbb{R}} \frac{\overline{\psi_{\lambda}}(\xi)}{\rho(\xi)} \widehat{\widehat{W}}(d \xi) \text { and } \mathbb{E}\left(d_{X}^{2}(a, .)\right)=\mathcal{I}_{1}(a)
$$


2. for all $i=0,1, \cdots, K$, if the scale a is such that $\left[\frac{\alpha}{a}, \frac{\beta}{a}\right] \subset\left[\omega_{i}, \omega_{i+1}\right]$, then

$$
\mathbb{E}\left(d_{X}^{2}(a, .)\right)=a^{2 H_{i}+1} \sigma_{i}^{2} K_{H_{i}}(\psi), \text { with } K_{H}(\psi)=\int_{\mathbb{R}} \frac{|\widehat{\psi}(u)|^{2}}{|u|^{2 H+1}} d u .
$$

Remark 3.1 Another possible interpretation of this property is the following: the process $\left(d_{X}(t, 0)\right)_{t \in \mathbb{R}_{+}}$ is a continuous centered Gaussian process $H_{i}$-self-similar for $t \in\left[\frac{\beta}{\omega_{i+1}}, \frac{\alpha}{\omega_{i}}\right]$, i.e. $\left(d_{X}(t, 0)\right)_{\beta / \omega_{i+1} \leq t \leq \alpha / \omega_{i}}$ is a $H_{i}$-self-similar process. However, such a process has no stationary increments.

The property (11) is useful for the estimation of the parameters of $X$. Indeed, we deduce that if the scale $a \in\left[\frac{\beta}{\omega_{i+1}}, \frac{\alpha}{\omega_{i}}\right]$, then

$$
\log \left(\mathbb{E}\left(d_{X}^{2}(a, .)\right)\right)=\left(2 H_{i}+1\right) \log a+\log \sigma_{i}^{2}+\log K_{H_{i}}(\psi) .
$$

Thus, if we consider a convergent estimator of $\log \left(\mathbb{E}\left(d_{X}^{2}(a,).\right)\right)$, it provides a linear model in $\log a$ and $\log \sigma_{i}^{2}$. This natural estimator is $\log I_{N}(a)$ with

$$
I_{N}(a)=\frac{1}{[N / a]-1} \sum_{k=1}^{[N / a]-1} d_{X}^{2}\left(a, k \Delta_{N}\right) .
$$

This choice is motivated by two arguments. First, we only observe the values $X(0), X\left(\Delta_{N}\right), \cdots, X\left(N \Delta_{N}\right)$, then the function $\psi$ is essentially supported around 0 . The stationarity of $d_{X}^{2}(a,$.$) implies that for$ all $a>0$,

$$
\mathbb{E}\left(I_{N}(a)\right)=\mathbb{E}\left(d_{X}^{2}(a, .)\right)
$$

With these notations, the main result of this paper, that is the following functional central limit theorem satisfied by $\left(\log I_{N}(a)\right)_{a_{\min } \leq a \leq a_{\max }}$, can be established:

Theorem 3.1 Let $X$ be $a\left(M_{K}\right)$-F.B.M., $0<a_{\min }<a_{\max }$ and $\psi$ satisfy Assumption (A1). Then,

$$
\sqrt{N \Delta_{N}}\left(\log I_{N}(a)-\log \mathcal{I}_{1}(a)\right)_{a_{\min } \leq a \leq a_{\max }} \underset{N \rightarrow \infty}{\stackrel{\mathcal{D}}{\longrightarrow}}(Z(a))_{a_{\min } \leq a \leq a_{\max }}
$$

with $(Z(a))$ a centered Gaussian process such that for $\left(a_{1}, a_{2}\right) \in\left[a_{\min }, a_{\max }\right]^{2}$,

$$
\operatorname{cov}\left(Z\left(a_{1}\right), Z\left(a_{2}\right)\right)=\frac{2 a_{1} a_{2}}{\mathcal{I}_{1}\left(a_{1}\right) \mathcal{I}_{1}\left(a_{2}\right)} \int_{\mathbb{R}}\left(\int_{\mathbb{R}} \frac{\overline{\widehat{\psi}}\left(a_{1} \xi\right) \widehat{\psi}\left(a_{2} \xi\right)}{|\rho(\xi)|^{2}} e^{-i u \xi} d \xi\right)^{2} d u .
$$

Then, if we specify the localization of scales, i.e. frequencies, we obtain the following consequence:

Corollary 3.1 Let $i \in\{0,1, \cdots, K\}$ and assume that $\frac{\beta}{\alpha} \leq \frac{\omega_{i+1}}{\omega_{i}}$. Then,

$$
\begin{aligned}
\sqrt{N \Delta_{N}}\left(\log I_{N}(1 / f)+\left(2 H_{i}+1\right) \log f-\log \sigma_{i}^{2}-\log K_{H_{i}}(\psi)\right)_{\omega_{i} / \alpha \leq f \leq \omega_{i+1} / \beta} \\
\underset{N \rightarrow \infty}{\stackrel{\mathcal{D}}{\longrightarrow}}(Z(1 / f))_{\omega_{i} / \alpha \leq f \leq \omega_{i+1} / \beta}
\end{aligned}
$$

with the centered Gaussian process $(Z()$.$) such that for \left(f_{1}, f_{2}\right) \in\left[\frac{\omega_{i}}{\alpha}, \frac{\omega_{i+1}}{\beta}\right]^{2}$,

$$
\operatorname{cov}\left(Z\left(1 / f_{1}\right), Z\left(1 / f_{2}\right)\right)=\frac{2\left(f_{1} f_{2}\right)^{2 H_{i}}}{K_{H_{i}}^{2}(\psi)} \int_{\mathbb{R}}\left(\int_{\mathbb{R}} \frac{\overline{\widehat{\psi}}\left(\xi / f_{1}\right) \widehat{\psi}\left(\xi / f_{2}\right)}{|\xi|^{2 H_{i}+1}} e^{-i u \xi} d \xi\right)^{2} d u
$$


For $\Delta_{N}$ small enough, this result shows that every parameters $H_{i}$ and $\sigma_{i}^{2}$ could be estimate by a linear regression of $\log I_{N}\left(1 / f_{j}\right)$ onto $\log f_{j}$, when the frequencies $\omega_{i}$ are known. Moreover, this central limit theorem shows that a graph of $\left(\log f, \log I_{N}(1 / f)\right)$ for $f>0$ exhibits different areas of asymptotic linearity when $X$ is a $\left(M_{K}\right)$-F.B.M. This will be illustrated in the following example.

Example 3.1 (A numerical simulation) Let $X$ be a $\left(M_{1}\right)$-F.B.M., with $\sigma_{0}=\sigma_{1}=5, H_{0}=0.6$, $H_{1}=0.2$ and $\omega_{1}=0.5$. A discretized trajectory $\left(X\left(\Delta_{N}\right), \cdots, X\left(N . \Delta_{N}\right)\right.$ ) (where $N=3000$ and $\Delta_{N}=0.05$ ) is numerically obtained from property (3) and Cholesky method which provides L.U. decomposition of the covariance matrix of $\left(X\left(\Delta_{N}\right), \cdots, X\left(N . \Delta_{N}\right)\right)$.

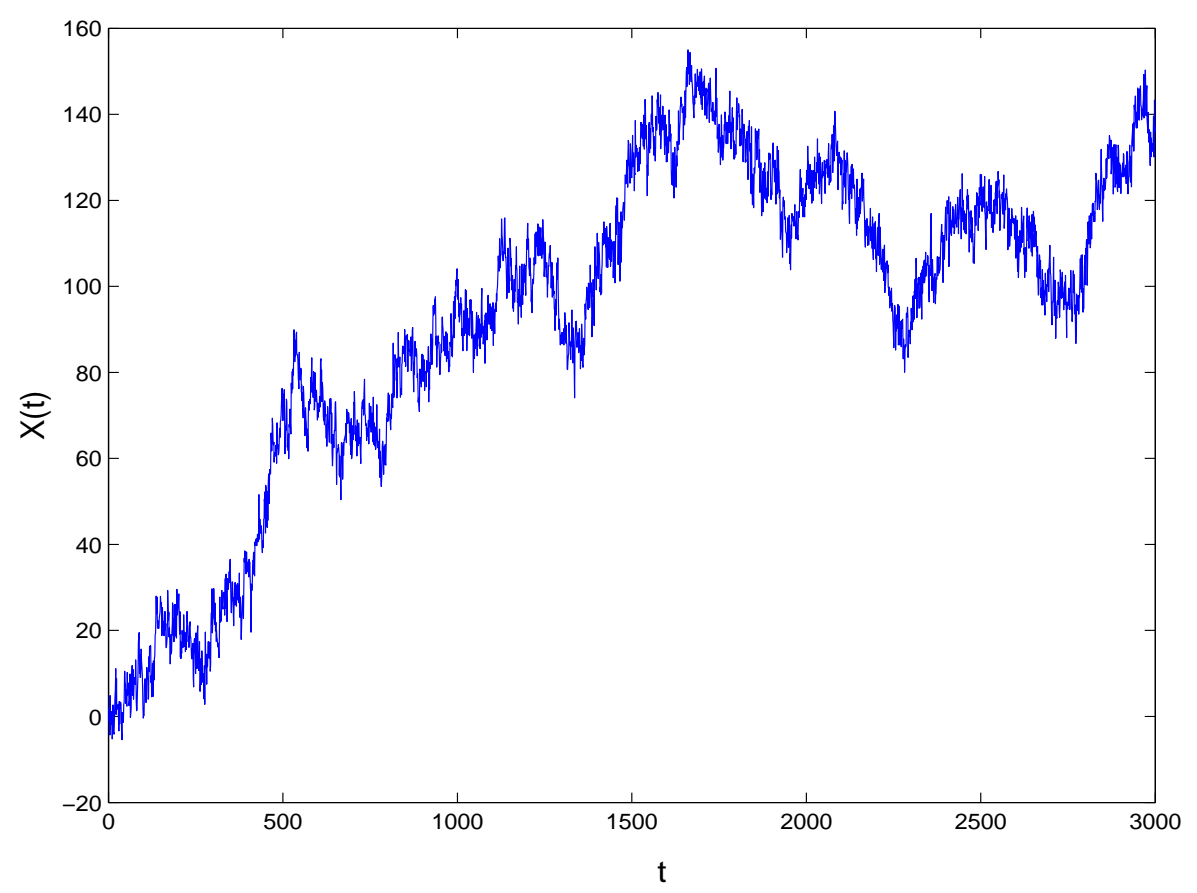

Figure 1: A discretized path $(X(0.05), X(0.10), \cdots, X(150))$ of a $\left(M_{1}\right)$-F.B.M., with $\sigma_{0}=\sigma_{1}=5$, $H_{0}=0.6, H_{1}=0.2$ and $\omega_{1}=0.5$.

Now it is possible to compute $\left(\log I_{N}(f), \log f\right)$ for numerous values of $f$. More precisely, we define $f_{i}=f_{m} q^{i / 150}$ for $i=0,1, \cdots, 150$ such that $q=\frac{f_{M}}{f_{m}}$ with $f_{m}=0.01$ and $f_{M}=10$. The chosen mother wavelet is the Lemarié-Meyer wavelet with $\alpha=2 \pi / 3$ and $\beta=8 \pi / 3$. 


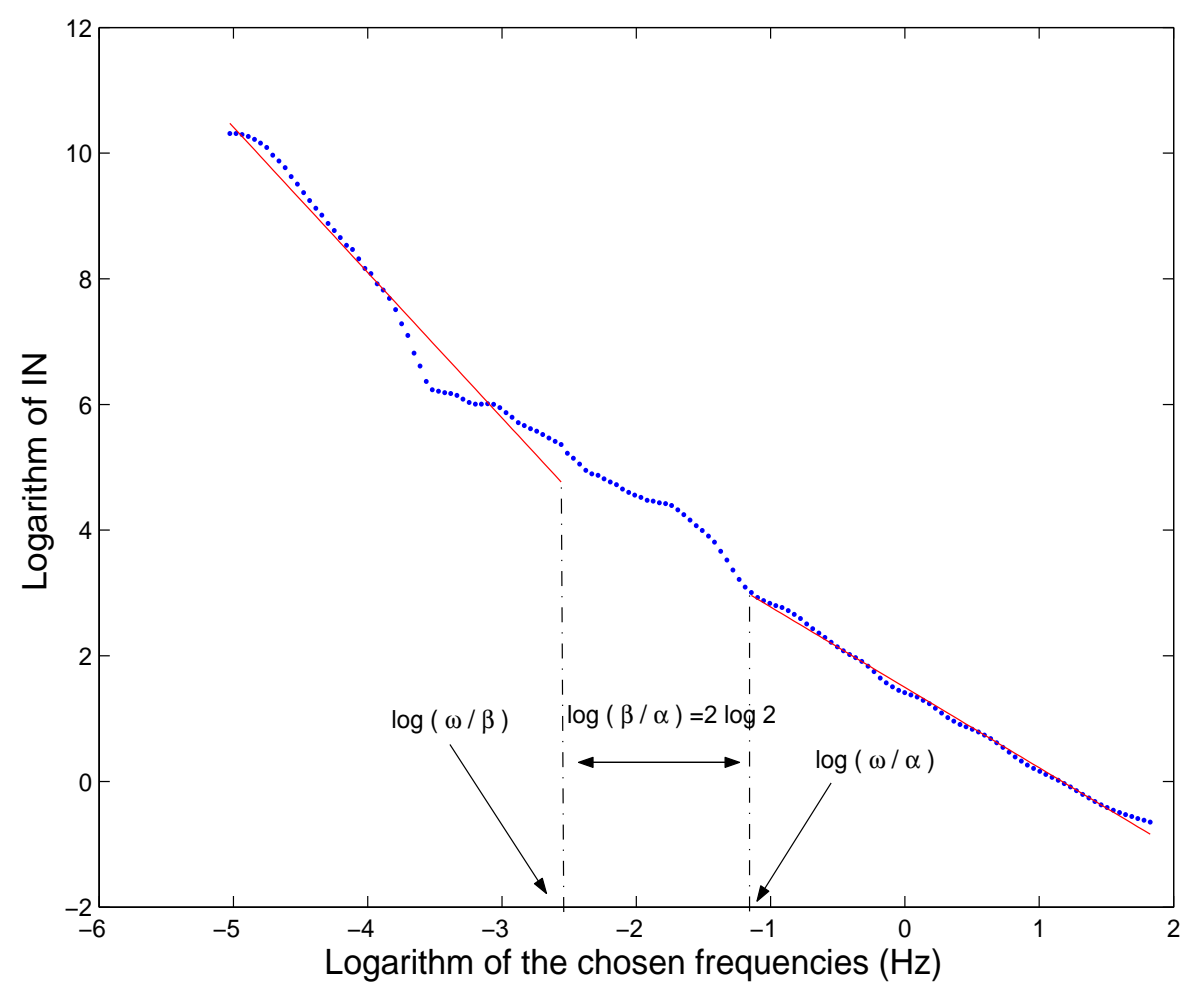

Figure 2: The double logarithm plotting of the variance of wavelet coefficients and its corresponding regression lines for the previous path of a $\left(M_{1}\right)$-F.B.M.

We observe both the different intervals of linearity and obtain the following estimations by linear regression: $\widehat{H}_{0} \simeq 0.16$ and $\widehat{H}_{1} \simeq 0.65$ that are rather good estimations. This was done with the knowledge of $\omega_{1}$. In Bardet and Bertrand (2003), we develop a method for estimating $\left(\omega_{i}\right)_{i}$, the different parameters $\left(H_{i}\right)_{i}$ and $\left(\sigma_{i}^{2}\right)_{i}$ and construct a goodness-of-fit test for a $\left(M_{K}\right)$-F.B.M.

\section{A Two examples of applications}

\section{A.1 Finance}

Numerous papers in financial economic exhibit the long-range dependence of the price of shares. They are based on statistical studies of financial data, see for instance Willinger et al. (1999) and the references therein. These studies have suggested the modelling of log-price process by a fractional Brownian motion. But Rogers (1997) has shown that the F.B.M. is an absurd candidate for the $\log$-price of a share, say $X$, since modelling by F.B.M. $\left(X=B_{H}\right)$ would induce the existence of arbitrage opportunities. This mainly follows from the fact that a F.B.M. $B_{H}$ is not a semimartingale except when $H=1 / 2$. Anyway, the notions should be specify. To be exact, we say that an arbitrage exists if there is some trading strategy whose gain process $\left(Y_{t}\right)_{0 \leq t \leq 1}$ satisfies a) $Y_{0}=0 \leq Y_{1}$, b) $Y_{t} \geq-1$ for all $t \in[0,1]$, and c) $\mathbb{P}\left(Y_{1}>0\right)>0$. If the log-price process is not a semi-martingale then there can be no equivalent probability (called risk neutral probability) under which $X$ becomes local martingale. The existence of (at least) one risk neutral probability 
is equivalent to the NFLVR condition (= No Free Lunch at Vanishing Risk), see Delbaen and Schachermayer (1994). The existence of an arbitrage implies the existence of a FLVR, but the converse is false, see counterexamples in Delbaen and Schachermayer (1994). Rogers (1997) shows that when $H \neq 1 / 2$

1. the F.B.M. $B_{H}$ is not a semi-martingale, then there exists no risk neutral probability,

2. there exists an arbitrage.

This study is based on the moving average representation

$$
X_{\varphi}(t)=C_{\varphi}^{-1} \int_{-\infty}^{+\infty}[\varphi(t-s)-\varphi(-s)] d W_{s}
$$

with the kernel $\varphi(x)=|x|^{H-1 / 2} \mathbf{1}_{[0,+\infty}[(x)$. But Rogers (1997) remarked that the existence of arbitrage is linked to the behaviour of the kernel at the vicinity of 0 . He proposed to replace the kernel by a regularized kernel (in the vicinity of 0 ), then the process $X$ defined by (18) becomes a semi-martingale and exhibit the same long-range dependence as the increments of the F.B.M. Cheridito (2000) gives a class of regularized kernel for which the corresponding price process is arbitrage-free and also equivalent to Brownian motion, therefore there exists a unique risk neutral probability and he derives formulas for the price of European option.

The regularization of the kernel $\varphi$ near 0 corresponds to a modification of the high frequencies behaviour of the process. The case where $X$ is a $\left(M_{K}\right)$-F.B.M. corresponds to another modification. Formula (4) shows that a $\left(M_{K}\right)$-F.B.M. is a semi-martingale if and only if $H_{K}=1 / 2$. Let us specify the reasoning, $X$ is a semi-martingale if and only if $B_{H_{K}}$ is a semi-martingale and the F.B.M. $B_{H_{K}}$ is a semi-martingale if and only if $H_{K}=1 / 2$, see Rogers (1997). In conclusion, the $\left(M_{K}\right)$-F.B.M. with $H_{K}=1 / 2$ appears as a reasonable model for the log-price process, this is confirm by the statistical study given in Bardet (2000). The pricing of European option with this model remains open.

\section{A.2 Biomechanical applications}

In Biomechanic, one wishes to model the upright position of human being in quiet stance and understand the control system maintaining the upright posture. The position of the center of pressure (C.O.P.) is measured on a force platform during one minute at $100 \mathrm{~Hz}$, leading to set of 6000 data. Collins and de Luca (1993) [16] introduced the use of F.B.M. for modelling these data. From the application of the log-variogram method, Collins and de Luca interpret the obtained graph as corresponding to a F.B.M. with two regimes a short term with slope $2 H_{1}$ and a long term with slope $2 H_{0}$ separated by a critical time lag $\delta_{c}$ and these parameters are estimated graphically: one obtains $H_{0}>0.5, H_{1}<0.5$ and a critical time lag $\delta_{c} \simeq 1 \mathrm{~s}$. In fact, the first analysis would suggest the modelling by a $\left(M_{1}\right)$-F.B.M. This method has been used many times in biomechanics with different experimental conditions (opened eyes versus closed eyes, different feet angles,...). But the automatic determination of the critical time lag $\delta_{c}$ (corresponding to the frequency change $\omega_{1}$ ) and of the two 
slopes $2 H_{0}, 2 H_{1}$ is problematic with the statistic based on the variogram.

Here, we present the result of the wavelet analysis for a lateral path of the C.O.P. The chosen mother wavelet is such that $\alpha=5$ and $\beta=10$ and the frequency change is automatically detected. We refer to Bardet and Bertrand (2003) for a complete study of biomechanical data and its conclusions.

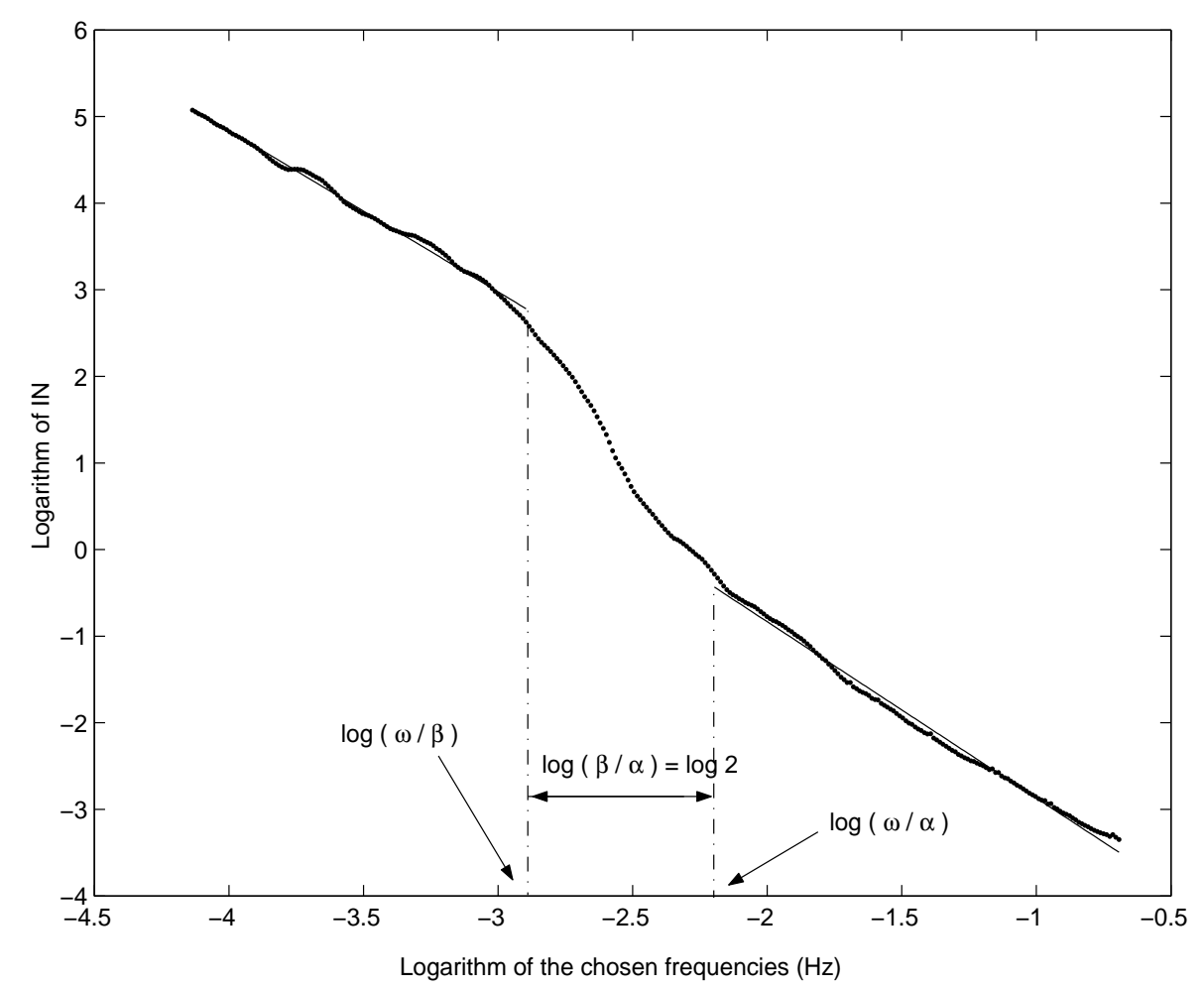

Figure 2: The double logarithm plotting of the variance of wavelet coefficients and its corresponding regression lines for a lateral trajectory of the C.O.P. ${ }^{1}$

\section{B Proofs}

\section{B.1 Proofs of section 2}

Proof. [Property 2.1] 1. See Cramér and Leadbetter (1967) for the proof of the stationarity of the increments of $X$.

2. We easily obtain:

$$
\begin{aligned}
\mathcal{V}(\delta) & =\int_{\mathbb{R}} \frac{\left|e^{i \delta \xi}-1\right|^{2}}{\rho^{2}(\xi)} d \xi=4 \int_{0}^{+\infty} \frac{(1-\cos (\delta \xi))}{\rho^{2}(\xi)} d \xi \\
& =4 \sum_{i=0}^{K} \sigma_{i}^{2} \int_{\omega_{i}}^{\omega_{i+1}} \frac{(1-\cos (\delta \xi))}{\xi^{2 H_{i}+1}} d \xi
\end{aligned}
$$

\footnotetext{
${ }^{1}$ these experimental data were realized by A. Mouzat and are used in [13].
} 
that provides us (3) after the change of variable $\delta \xi=v$.

3. Formula (2) gives us

$$
\begin{aligned}
X(t) & =\int_{\mathbb{R}} \frac{\left(e^{i t \xi}-1\right)}{|\xi|^{H_{K}+1 / 2}} \widehat{W}(d \xi) \\
& -\int_{|\xi| \leq \omega_{K}} \frac{\left(e^{i t \xi}-1\right)}{|\xi|^{H_{K}+1 / 2}} \widehat{W}(d \xi)+\sum_{j=0}^{K-1} \int_{\omega_{j}<|\xi| \leq \omega_{j+1}} \frac{\left(e^{i t \xi}-1\right)}{|\xi|^{H_{j}+1 / 2}} \widehat{W}(d \xi)
\end{aligned}
$$

The harmonizable representation (1) shows that $\int_{\mathbb{R}} \frac{\left(e^{i t \xi}-1\right)}{|\xi|^{H_{K}+1 / 2}} \widehat{W}(d \xi)$ is a F.B.M. with Hurst index $H_{K}$, we call it $B_{H_{K}}$, this provides the decomposition (4) with

$$
R(t)=Z_{H_{K}, \omega_{K}}(t)+\sum_{j=0}^{K-1}\left(Z_{H_{j}, \omega_{j+1}}(t)-Z_{H_{j}, \omega_{j}}(t)\right)
$$

where by definition

$$
Z_{H, \omega}(t)=\int_{|\xi| \leq \omega} \frac{\left(e^{i t \xi}-1\right)}{|\xi|^{H+1 / 2}} \widehat{W}(d \xi)
$$

When $H \in] 0,1[$ and $\omega \in] 0,+\infty\left[\right.$, the process $Z_{H, \omega}$ has finite variation. Since $R(t)$ is a finite sum of these processes, it is a process with finite variation. Therefore, the decomposition (4) induces that the regularity of $X$ is exactly the regularity of the F.B.M. with parameter $B_{H_{K}}$. This finishes the proof of Property 2.1.

Proof. [Property 2.2] First, we have

$$
r(n)=8 \sum_{i=0}^{K} \sigma_{i}^{2} \int_{\omega_{i}}^{\omega_{i+1}} \frac{\cos (n \xi) \sin ^{2}(\xi / 2)}{\xi^{2 H_{i}+1}} d \xi .
$$

Indeed, using (2) and the definition of $r(n)$, we get:

$$
\begin{aligned}
r(n)=\operatorname{cov}(Y(n), Y(0)) & =\operatorname{cov}[(X(n+1)-X(n)),(X(1)-X(0))] \\
& =\int_{\mathbb{R}} \frac{\left(e^{i \xi(n+1)}-e^{i \xi n}\right)}{\rho(\xi)} \frac{\left(e^{-i \xi}-1\right)}{\rho(\xi)} d \xi \\
& =\int_{\mathbb{R}} e^{i n \xi} \frac{\left|e^{-i \xi}-1\right|^{2}}{\rho^{2}(\xi)} d \xi \\
& =\int_{0}^{+\infty} \frac{\cos (n \xi) \sin ^{2}(\xi / 2)}{\rho^{2}(\xi)} d \xi
\end{aligned}
$$

which induces (19) by definition of the $\left(M_{k}\right)$-F.B.M. Formulae (5) and (6) are asymptotic expansion of $r(n)$ following the powers of $1 / n$. After two integrations by parts, we have the following 
calculations: for every $\omega>0$ and $H \in] 0,1[$

$$
\begin{aligned}
\int_{\omega}^{\infty} & \frac{\cos (n \xi) \sin ^{2}(\xi / 2)}{\xi^{2 H+1}} d \xi= \\
= & \frac{1}{n}\left[\frac{-\sin (n \omega) \sin ^{2}(\omega / 2)}{\omega^{2 H+1}}-\int_{\omega}^{\infty} \sin (n \xi)\left(\frac{\sin \xi}{2 \xi^{2 H+1}}-\frac{(2 H+1) \sin ^{2}(\xi / 2)}{\xi^{2 H+2}}\right) d \xi\right] \\
= & -\frac{1}{n}\left(\frac{\sin (n \omega) \sin ^{2}(\omega / 2)}{\omega^{2 H+1}}\right)-\frac{1}{n^{2}} \cos (n \omega)\left(\frac{\sin \omega}{\omega^{2 H+1}}-(2 H+1) \frac{\sin ^{2}(\omega / 2)}{\omega^{2 H+2}}\right) \\
& +\frac{1}{n^{2}} \int_{\omega}^{\infty} \cos n \xi\left(\frac{\cos \xi}{\xi^{2 H+1}}-(2 H+1) \frac{\sin (\xi)}{\xi^{2 H+2}}+(2 H+1)(2 H+2) \frac{\sin ^{2}(\xi / 2)}{\xi^{2 H+2}}\right) d \xi
\end{aligned}
$$

For $\omega>0$ and $H>0$, the last integral is bounded by

$$
F \times \int_{\omega}^{\infty}\left[\xi^{-(2 H+1)}+\xi^{-(2 H+2)}\right] \leq F \times\left[\omega^{-2 H}+\omega^{-(2 H+1)}\right]<+\infty
$$

with $F>0$. This induces for every $\omega>0$ and $H \in] 0,1[$

$$
\int_{\omega}^{\infty} \frac{\cos (n \xi) \sin ^{2}(\xi / 2)}{\xi^{2 H+1}} d \xi=-\frac{1}{n}\left(\frac{\sin (n \omega) \sin ^{2}\left(\frac{\omega}{2}\right)}{\omega^{2 H+1}}\right)+\mathcal{O}\left(\frac{1}{n^{2}}\right) .
$$

We immediately deduce from (20) that for $i=1,2, . ., K$, with $\omega_{K+1}=\infty$, we have

$$
\begin{aligned}
\int_{\omega_{i}}^{\omega_{i+1}} \frac{\cos (n \xi) \sin ^{2}(\xi / 2)}{\xi^{2 H_{i}+1}} d \xi= \\
\quad=\frac{1}{n}\left(\frac{\sin \left(n \omega_{i+1}\right) \sin ^{2}\left(\frac{\omega_{i+1}}{2}\right)}{\omega_{i+1}^{2 H_{i}+1}}-\frac{\sin \left(n \omega_{i}\right) \sin ^{2}\left(\frac{\omega_{i}}{2}\right)}{\omega_{i}^{2 H_{i}+1}}\right)+\mathcal{O}\left(\frac{1}{n^{2}}\right) .
\end{aligned}
$$

From the other hand, consider the first term in the sum in the right hand side of (19). We have

$$
\begin{aligned}
\int_{0}^{\omega_{1}} \frac{\cos (n \xi) \sin ^{2}(\xi / 2)}{\xi^{2 H_{0}+1}} & \\
= & \int_{0}^{+\infty} \frac{\cos (n \xi) \sin ^{2}(\xi / 2)}{\xi^{2 H_{0}+1}} d \xi-\int_{\omega_{1}}^{+\infty} \frac{\cos (n \xi) \sin ^{2}(\xi / 2)}{\xi^{2 H_{0}+1}} d \xi .
\end{aligned}
$$

The first integral correspond to the case of a constant Hurst parameter, that is the usual F.B.M. Let $X_{0}$ be a F.B.M. with parameter $H_{0}$ and variance 1 , defined by

$$
X_{0}(t)=\frac{1}{C\left(H_{0}\right)} \int_{\mathbb{R}} \frac{\left(e^{i t \xi}-1\right)}{|\xi|^{H_{0}+1 / 2}} \widehat{\widehat{W}}(d \xi) \text { for } t \in \mathbb{R}_{+}
$$

with $C\left(H_{0}\right)=\pi^{1 / 2}\left(H_{0} \Gamma\left(2 H_{0}\right) \sin \left(\pi H_{0}\right)\right)^{-1 / 2}$, then we have

$$
\operatorname{cov}\left(\left(X_{0}(n+1)-X_{0}(n), X_{0}(1)-X_{0}(0)\right)=\frac{1}{C^{2}\left(H_{0}\right)} \int_{0}^{\infty} \frac{\cos (n \xi) \sin ^{2}(\xi / 2)}{\xi^{2 H_{0}+1}} d \xi\right.
$$

and (see [29] p. 335) we have the following expansion

$$
\operatorname{cov}\left(\left(X_{0}(n+1)-X_{0}(n), X_{0}(1)-X_{0}(0)\right)=H_{0}\left(2 H_{0}-1\right) \frac{1}{n^{2-2 H_{0}}}+\mathcal{O}\left(\frac{1}{n^{3-2 H_{0}}}\right) .\right.
$$


Combined with (20) and (22), for all $\left.H_{0} \in\right] 0,1[$, we have

$$
\begin{aligned}
& \int_{0}^{\omega_{1}} \frac{\cos (n \xi) \sin ^{2}(\xi / 2)}{\xi^{2 H_{0}+1}} d \xi= \\
& \quad=\left(\frac{\pi\left(2 H_{0}-1\right)}{r\left(2 H_{0}\right) \sin \left(\pi H_{0}\right)}\right) \frac{1}{n^{2-2 H_{0}}}+\left(\frac{\sin \left(n \omega_{1}\right) \sin ^{2}\left(\frac{\omega_{1}}{2}\right)}{\omega_{1}^{2 H_{0}+1}}\right) \frac{1}{n}+\mathcal{O}\left(\frac{1}{n^{2}}+\frac{1}{n^{3-2 H_{0}}}\right) .
\end{aligned}
$$

Therefore from (21) and (23), for $H_{0}>1 / 2$, we obtain (5) and if $0<H_{0} \leq 1 / 2$,

$$
\begin{aligned}
\operatorname{cov} & (Y(n) Y(0)) \\
=\frac{8}{n} & {\left[\sum_{i=1}^{K-1} \sigma_{i}^{2}\left(\frac{\sin \left(n \omega_{i+1}\right) \sin ^{2}\left(\frac{\omega_{i+1}}{2}\right)}{\omega_{i+1}^{1+2 H_{i+1}}}-\frac{\sin \left(n \omega_{i}\right) \sin ^{2}\left(\frac{\omega_{i}}{2}\right)}{\omega_{i}^{1+2 H_{i}}}\right)\right.} \\
& \left.+\sigma_{0}^{2}\left(\frac{\sin \left(n \omega_{1}\right) \sin ^{2}\left(\frac{\omega_{1}}{2}\right)}{\omega_{1}^{2 H_{0}+1}}\right)-\sigma_{K}^{2} \frac{\sin \left(n \omega_{K}\right) \sin ^{2}\left(\frac{\omega_{K}}{2}\right)}{\omega_{K}^{2 H_{K}+1}}\right] \\
& +\frac{\pi \sigma_{0}^{2}\left(2 H_{0}-1\right)}{\Gamma\left(2 H_{0}\right) \sin \left(\pi H_{0}\right)} \frac{1}{n^{2-2 H_{0}}}+\mathcal{O}\left(\frac{1}{n^{2}}\right),
\end{aligned}
$$

and (6) is proved.

\section{B.2 Proofs of section 3}

Proof. [Property 3.1] First we have

$$
\begin{aligned}
d_{X}(\lambda) & =\int_{\mathbb{R}} \psi_{\lambda}(t)\left[\int_{\mathbb{R}} \frac{\left(e^{i t \xi}-1\right)}{\rho(\xi)} \overline{\widehat{W}}(d \xi)\right] d t \\
& =\int_{\mathbb{R}} \frac{1}{\rho(\xi)}\left[\int_{\mathbb{R}}\left(e^{i t \xi}-1\right) \psi_{\lambda}(t) d t\right] \widehat{\widehat{W}}(d \xi)
\end{aligned}
$$

But $\psi$ satisfies $\int \psi=0$ and it implies (10). Now, we prove that $\left(d_{X}(a, b)\right)_{b \in \mathbb{R}}$ is a stationary centered Gaussian process. The only problem is the stationarity. It is obvious that

$$
\overline{\widehat{\psi_{\lambda}}}(\xi)=\sqrt{a} \times e^{-i a b \xi} \times \overline{\widehat{\psi}}(a \xi)
$$

Combined with (10), we deduce that for $a>0$ and $\left(b, b^{\prime}\right) \in \mathbb{R}^{2}$, we have:

$$
\mathbb{E}\left(d_{X}(a, b) d_{X}\left(a, b^{\prime}\right)\right)=\sqrt{a a^{\prime}} \int_{\mathbb{R}} e^{-i a\left(b-b^{\prime}\right) \xi}\left|\frac{\widehat{\psi}(a \xi)}{\rho(\xi)}\right|^{2} d \xi
$$

Thus, for a given $a>0, \mathbb{E}\left(d_{X}(a, b) d_{X}\left(a, b^{\prime}\right)\right)$ is only depending on $\left(b-b^{\prime}\right)$ which induces that $\left(d_{X}(a, b)\right)_{b \in \mathbb{R}}$ is a stationary process.

The second part of the property is a consequence of this first part. In fact, when $b=b^{\prime}$, the scale $a$ satisfies $\left.\left[\frac{\alpha}{a}, \frac{\beta}{a}\right] \subset\right] \omega_{i}, \omega_{i+1}[$ and $\psi$ satisfies Assumption (A1), formula (25) implies

$$
\mathbb{E}\left(d_{X}^{2}(a, .)\right)=2 a \int_{\alpha / a}^{\beta / a}\left|\frac{\bar{\psi}(a v)}{\rho(v)}\right|^{2} d v
$$




$$
\begin{aligned}
& =2 a \int_{\alpha / a}^{\beta / a}|\widehat{\psi}(a v)|^{2} \sigma_{i}^{2} v^{-\left(2 H_{i}+1\right)} d v \\
& =a \sigma_{i}^{2} \int_{\mathbb{R}}|\widehat{\psi}(a v)|^{2} v^{-\left(2 H_{i}+1\right)} d v \\
& =a^{2 H_{i}+1} \sigma_{i}^{2} K_{H_{i}}(\psi),
\end{aligned}
$$

and the second part of the property is proved.

Proof. [Theorem 3.1] (Both first steps follow the same procedure that in Bardet, 2002).

First step: For $\left.\left(a, a^{\prime}\right) \in\right] 0, \infty\left[{ }^{2}\right.$, and $\left(b, b^{\prime}\right) \in \mathbb{R}^{2}$, using (24) we get:

$$
\begin{aligned}
\left|\mathbb{E}\left(d_{X}(a, b) d_{X}\left(a^{\prime}, b^{\prime}\right)\right)\right| & =\sqrt{a a^{\prime}} \int_{\mathbb{R}} e^{-i\left(a b-a^{\prime} b^{\prime}\right) \xi} \frac{\overline{\widehat{\psi}}(a \xi) \widehat{\psi}\left(a^{\prime} \xi\right)}{|\rho(\xi)|^{2}} d \xi \\
& \leq \frac{C}{\left(a b-a^{\prime} b^{\prime}\right)^{2}},
\end{aligned}
$$

when $\left|a b-a^{\prime} b^{\prime}\right|>1$. The last inequality follows from assumption (A1) and a double integration by part (with $C>0$ ). By this way, and with:

$$
\begin{aligned}
\operatorname{cov}\left(I_{N}(a) I_{N}\left(a^{\prime}\right)\right) & =\frac{1}{[N / a]\left[N / a^{\prime}\right]} \sum_{p=1}^{[N / a]} \sum_{p^{\prime}=1}^{\left[N / a^{\prime}\right]} \operatorname{cov}\left(d_{X}^{2}\left(a, p \Delta_{N}\right) d_{X}^{2}\left(a^{\prime}, p^{\prime} \Delta_{N}\right)\right) \\
& =\frac{2}{[N / a]\left[N / a^{\prime}\right]} \sum_{p=1}^{[N / a]} \sum_{p^{\prime}=1}^{\left[N / a^{\prime}\right]} \operatorname{cov}^{2}\left(d_{X}\left(a, p \Delta_{N}\right) d_{X}\left(a^{\prime}, p^{\prime} \Delta_{N}\right)\right),
\end{aligned}
$$

because $d_{X}(.,$.$) is a Gaussian random variable. Define \lambda_{N}\left(a, a^{\prime}\right)$ the set of pair $\left(p, p^{\prime}\right)$ such that $\left|a p \Delta_{N}-a^{\prime} p^{\prime} \Delta_{N}\right|<1$, then for $\left(p, p^{\prime}\right) \in \lambda_{N}\left(a, a^{\prime}\right)$ we have

$$
\operatorname{cov}^{2}\left(d_{X}\left(a, p \Delta_{N}\right) d_{X}\left(a^{\prime}, p^{\prime} \Delta_{N}\right)\right) \leq \mathbb{E} d_{X}^{2}(a, .) \mathbb{E} d_{X}^{2}\left(a^{\prime}, .\right) \leq M\left(a, a^{\prime}\right)
$$

with $M\left(a, a^{\prime}\right)>0$. From the other hand, for a pair $\left(p, p^{\prime}\right)$ satisfying $\left|a p \Delta_{N}-a^{\prime} p^{\prime} \Delta_{N}\right| \geq 1$, formula (26) induces that

$$
\operatorname{cov}^{2}\left(d_{X}\left(a, p \Delta_{N}\right) d_{X}\left(a^{\prime}, p^{\prime} \Delta_{N}\right)\right) \leq C^{2}\left|a p \Delta_{N}-a^{\prime} p^{\prime} \Delta_{N}\right|^{-4}
$$

Second step: we get

$$
\begin{aligned}
\operatorname{cov}\left(I_{N}(a) I_{N}\left(a^{\prime}\right)\right) & =\frac{2}{[N / a]\left[N / a^{\prime}\right]}\left(\sum_{\left(p, p^{\prime}\right) \in \lambda_{N}\left(a, a^{\prime}\right)} \operatorname{cov}^{2}\left(d_{X}^{2}\left(a, p \Delta_{N}\right) d_{X}^{2}\left(a^{\prime}, p^{\prime} \Delta_{N}\right)\right)+. .\right. \\
& \left..+\sum_{\left(p, p^{\prime}\right) \notin \lambda_{N}\left(a, a^{\prime}\right)} \operatorname{cov}^{2}\left(d_{X}^{2}\left(a, p \Delta_{N}\right) d_{X}^{2}\left(a^{\prime}, p^{\prime} \Delta_{N}\right)\right)\right) \\
& \leq \frac{2}{[N / a]\left[N / a^{\prime}\right]}\left(M\left|\lambda_{N}\left(a, a^{\prime}\right)\right|+\frac{C^{\prime}}{\Delta_{N}^{2}} \iint_{\left|a t-a^{\prime} t^{\prime}\right| \geq 1} \frac{\mathbf{1}_{0 \leq \Delta_{N} t \leq N / a} \mathbf{1}_{0 \leq \Delta_{N} t^{\prime} \leq N / a^{\prime}}\left|a t-a^{\prime} t^{\prime}\right|^{4}}{\left[t d t^{\prime}\right)}\right. \\
& \leq \frac{2}{[N / a]\left[N / a^{\prime}\right]}\left(\frac{2 M N}{a a^{\prime} \Delta_{N}}+\frac{C^{\prime}}{\Delta_{N}^{2} a} \int_{0}^{\Delta_{N} N / a^{\prime}} \int_{1}^{N} \frac{d u}{u^{4}} d t^{\prime}\right) \\
& \leq \frac{M^{\prime}}{N \Delta_{N}}+\frac{C^{\prime \prime}}{N \Delta_{N}} .
\end{aligned}
$$


This induces that $N \Delta_{N} \operatorname{cov}\left(I_{N}(a) I_{N}\left(a^{\prime}\right)\right) \rightarrow \gamma_{\rho}\left(a, a^{\prime}\right)$ when $N \rightarrow \infty$, with $\gamma_{\rho}\left(a, a^{\prime}\right)>0$. More precisely, let $f\left(t, t^{\prime}\right)=\int_{\mathbb{R}} \frac{\overline{\widehat{\psi}}(a \xi) \widehat{\psi}\left(a^{\prime} \xi\right)}{|\rho(\xi)|^{2}} e^{-i\left(a t-a^{\prime} t^{\prime}\right) \Delta_{N} \xi} d \xi$. Then, $f^{2} \in \mathcal{C}^{\infty}\left(\mathbb{R}^{2}\right)$ because $\psi$ satisfies Assumption (A1). More, for $\left(k, k^{\prime}\right) \in \mathbb{N}^{*} \times \mathbb{N}^{*}$,

$$
\left|\int_{k-1}^{k} \int_{k^{\prime}-1}^{k^{\prime}}\left(f^{2}\left(t, t^{\prime}\right)-f^{2}\left(k, k^{\prime}\right)\right) d t d t^{\prime}\right| \leq 2 \sup _{\left(t, t^{\prime}\right) \in \mathbb{R}^{2}}\left|\left(\frac{\partial f}{\partial t}+\frac{\partial f}{\partial t^{\prime}}\right)\left(t, t^{\prime}\right)\right| \mid f\left(\theta_{k}, \theta_{k^{\prime}} \mid,\right.
$$

with $k-1 \leq \theta_{k} \leq k$ and $k^{\prime}-1 \leq \theta_{k^{\prime}} \leq k^{\prime}$. From obvious computations, it appears that $2 \sup _{\left(t, t^{\prime}\right) \in \mathbb{R}^{2}}\left|\left(\frac{\partial f}{\partial t}+\frac{\partial \bar{f}}{\partial t^{\prime}}\right)\left(t, t^{\prime}\right)\right| \leq C \Delta_{N}$ with $C$ a positive constant depending only on $\psi, \rho, a_{\text {min }}$ and $a_{\text {max }}$. Moreover, from the previous calculations $\frac{2 a a^{\prime} \Delta_{N}}{N} \sum_{p=1}^{[N / a]} \sum_{p^{\prime}=1}^{\left[N / a^{\prime}\right]} \mid f\left(\theta_{p}, \theta_{p^{\prime}} \mid<C^{\prime}\right.$. Therefore,

$$
\begin{aligned}
& N \Delta_{N} \operatorname{cov}\left(I_{N}(a) I_{N}\left(a^{\prime}\right)\right)=\frac{2 a a^{\prime} \Delta_{N}}{N} \sum_{p=1}^{[N / a]} \sum_{p^{\prime}=1}^{\left[N / a^{\prime}\right]}\left(\sqrt{a a^{\prime}} \int_{\mathbb{R}} \frac{\overline{\widehat{\psi}}(a \xi) \widehat{\psi}\left(a^{\prime} \xi\right)}{|\rho(\xi)|^{2}} e^{-i\left(a p-a^{\prime} p^{\prime}\right) \Delta_{N} \xi} d \xi\right)^{2} \\
& =\frac{2 a^{2} a^{\prime 2}}{N \Delta_{N}} \int_{t=0}^{[N / a] \Delta_{N}} \int_{t^{\prime}=0}^{\left[N / a^{\prime}\right] \Delta_{N}}\left(\int_{\mathbb{R}} \frac{\bar{\psi}(a \xi) \widehat{\psi}\left(a^{\prime} \xi\right)}{|\rho(\xi)|^{2}} e^{-i\left(a t-a^{\prime} t^{\prime}\right) \xi} d \xi\right)^{2} d t d t^{\prime}+\mathcal{O}\left(\Delta_{N}\right)
\end{aligned}
$$

Thus,

$$
\begin{aligned}
\gamma_{\rho}\left(a, a^{\prime}\right) & =\lim _{N \rightarrow \infty} N \Delta_{N} \operatorname{cov}\left(I_{N}(a) I_{N}\left(a^{\prime}\right)\right) \\
& =\lim _{N \rightarrow \infty} \frac{2 a^{2} a^{\prime 2}}{N \Delta_{N}} \int_{t=0}^{[N / a] \Delta_{N}} \int_{t^{\prime}=0}^{\left[N / a^{\prime}\right] \Delta_{N}}\left(\int_{\mathbb{R}} \frac{\overline{\widehat{\psi}}(a \xi) \widehat{\psi}\left(a^{\prime} \xi\right)}{|\rho(\xi)|^{2}} e^{-i\left(a t-a^{\prime} t^{\prime}\right) \xi} d \xi\right)^{2} d t d t^{\prime} \\
& =\lim _{N \rightarrow \infty} \frac{2 a a^{\prime}}{N \Delta_{N}} \int_{0}^{a[N / a] \Delta_{N}} \int_{0}^{a^{\prime}\left[N / a^{\prime}\right] \Delta_{N}}\left(\int_{\mathbb{R}} \frac{\overline{\widehat{\psi}}(a \xi) \widehat{\psi}\left(a^{\prime} \xi\right)}{|\rho(\xi)|^{2}} e^{-i\left(t-t^{\prime}\right) \xi} d \xi\right)^{2} d t d t^{\prime} \\
& =\lim _{N \rightarrow \infty} \frac{2 a a^{\prime}}{N \Delta_{N}} \int_{-N \Delta_{N}}^{N \Delta_{N}}\left(N \Delta_{N}-|u|\right)\left(\int_{\mathbb{R}} \frac{\overline{\widehat{\psi}}(a \xi) \widehat{\psi}\left(a^{\prime} \xi\right)}{|\rho(\xi)|^{2}} e^{-i u \xi} d \xi\right)^{2} d u \\
& =\lim _{N \rightarrow \infty} \frac{2 a a^{\prime}}{N \Delta_{N}}\left[\int_{|u| \leq N \Delta_{N}}\left(N \Delta_{N}\right)\left(\int_{\mathbb{R}} \frac{\overline{\widehat{\psi}}(a \xi) \widehat{\psi}\left(a^{\prime} \xi\right)}{|\rho(\xi)|^{2}} e^{-i u \xi} d \xi\right)^{2}+\cdots\right. \\
& \left.\left.\ldots+\int_{|u| \leq N \Delta_{N}}(-|u|)\left(\int_{\mathbb{R}} \frac{\overline{\widehat{\psi}}(a \xi) \widehat{\psi}\left(a^{\prime} \xi\right)}{|\rho(\xi)|^{2}} e^{-i u \xi} d \xi\right)^{2} d u\right]^{2}\right] \\
& =2 a a^{\prime} \int_{\mathbb{R}}\left(\int_{\mathbb{R}} \frac{\overline{\widehat{\psi}}(a \xi) \widehat{\psi}\left(a^{\prime} \xi\right)}{|\rho(\xi)|^{2}} e^{-i u \xi} d \xi\right)^{2} d u+\lim _{N \rightarrow \infty} \frac{2 a a^{\prime}}{N \Delta_{N}} \mathcal{O}(1),
\end{aligned}
$$

from the previous bound of $\gamma_{\rho}\left(a, a^{\prime}\right)>0$. Therefore, we obtain the following central limit theorem for all $m \in \mathbb{N}^{*}$ and $\left(a_{j}\right)_{1 \leq m} \in\left[a_{\min }, a_{\max }\right]^{m}$ :

$$
\sqrt{N \Delta_{N}}\left(I_{N}\left(a_{j}\right)-a_{j} \int_{\mathbb{R}}\left|\frac{\bar{\psi}\left(a_{j} \xi\right)}{\rho(\xi)}\right|^{2} d \xi\right)_{1 \leq j \leq m} \underset{N \rightarrow \infty}{\stackrel{\mathcal{D}}{\longrightarrow}} \mathcal{N}_{m}(0, \Gamma)
$$


with $\Gamma=\left(\gamma_{k, l}\right)_{1 \leq k, l \leq m}$ and

$$
\gamma_{k, l}=2 a_{k} a_{l} \int_{\mathbb{R}}\left(\int_{\mathbb{R}} \frac{\overline{\widehat{\psi}}\left(a_{k} \xi\right) \widehat{\psi}\left(a_{l} \xi\right)}{|\rho(\xi)|^{2}} e^{-i u \xi} d \xi\right)^{2} d u .
$$

Third step: Now, we have to prove the tightness of $\sqrt{N \Delta_{N}}\left(I_{N}(a)-\mathbb{E} I_{N}(a)\right)_{a_{\min } \leq a \leq a_{\max }}$. Let $a_{\max } \leq a_{1}<a<a_{2} \leq a_{\min }$ and $L_{N}(a)=\sqrt{N \Delta_{N}}\left(I_{N}(a)-\mathbb{E} I_{N}(a)\right)$. Then,

$$
\begin{aligned}
\mathbb{E}\left|L_{N}(a)-L_{N}\left(a_{1}\right)\right|^{2} & =N \Delta_{N}\left(\operatorname{var} I_{N}(a)+\operatorname{var} I_{N}\left(a_{1}\right)-2 \operatorname{cov}\left(I_{n}(a), I_{N}\left(a_{1}\right)\right)\right) \\
& =\gamma_{\rho}(a, a)+\gamma_{\rho}\left(a_{1}, a_{1}\right)-2 \gamma_{\rho}\left(a, a_{1}\right)+\frac{C^{\prime \prime}\left(N, a, a_{1}\right)}{N \Delta_{N}},
\end{aligned}
$$

from (27) and with $\left|C^{\prime \prime}\left(N, a, a_{1}\right)\right| \leq C\left(\psi, \rho, a_{\min }, a_{\max }\right)$ (see previously). But $\left(a, a_{1}\right) \mapsto \gamma_{\rho}\left(a, a_{1}\right)$ is $\mathcal{C}^{\infty}\left(\left[a_{\min }, a_{\text {max }}\right]^{2}\right)$ because $\psi$ satisfies Assumption (A1). Then, for $N$ large enough it exists a positive constant $D$, independent of $a$ and $a_{1}$ such that:

$$
\mathbb{E}\left|L_{N}(a)-L_{N}\left(a_{1}\right)\right|^{2} \leq D\left|a-a_{1}\right| .
$$

Therefore, using Cauchy-Schwartz inequality, we get

$$
\mathbb{E}\left|L_{N}(a)-L_{N}\left(a_{1}\right)\right|\left|L_{N}\left(a_{2}\right)-L_{N}(a)\right| \leq D \sqrt{\left|a-a_{1}\right|\left|a_{2}-a\right|},
$$

and thus the random function $L_{N}(a), N=1,2, \cdots$, of $\mathcal{D}\left(\left[a_{\min }, a_{\max }\right]\right)$ are tight by Theorem 15.4 and 15.6 of Billingsley (1968).

Fourth step: The function $x \mapsto \log x$ is $\mathcal{C}^{\infty}$ on $\mathbb{R}_{+}^{*}$. Thus, from the Delta-method theorem, Theorem 3.1 is proved.

Acknowledgment: We would like to thank the anonymous referee for all his valuable comments which have helped us to improve the earlier version of our work.

\section{References}

[1] Abry, P., Flandrin, P., Taqqu, M. and Veitch, D. , 2002. Self-similarity and long-range dependence through the wavelet lens, in Long-range Dependence: Theory and Applications, P. Doukhan, G. Oppenheim and M.S. Taqqu editors, Birkhäuser, p. 527-556..

[2] Ayache, A., Bertrand, P. and Lévy Véhel J. , 2006, "A central limit theorem for the generalized quadratic variations of the step fractional Brownian motion", to appear in Statistical Inference for Stochastic Processes.

[3] Ayache, A. and Lévy Véhel, J., "The Generalized Multifractional Brownian Motion." Statistical Inference for Stochastic Processes, Vol. 3, Issue 1/2, p. 7-18, 2000.

[4] Ayache, A. and Lévy Véhel, J., 2004, "On the identification of the pointwise Hölder exponent of the generalized multifractional Brownian motion." Stochastic Process. Appl., 111, p. 119-156. 
[5] Bardet J.M., 2000. "Les cours d'actifs financiers sont-ils autosimilaires?" Journal de la SFdS 141, p. 137-148.

[6] Bardet J.M., 2002. "Statistical study of the wavelet analysis of fractional Brownian motion." IEEE Trans. Inform. Theory. 48, p. 991-999.

[7] Bardet J.M. and Bertrand, P. "Identification of the multiscale fractional Brownian motion with biomechanical applications", to appear in Journal of Time Series Analysis.

[8] Bardet J.M., Lang G., Moulines E. and Soulier P., 2000. "Wavelet estimator of long-range dependent processes." Statistical Inference for Stochastic Processes 3, p. 85-99.

[9] Benassi, A. , Bertrand, P., Cohen, S., and Istas, J. "Identification of the Hurst index of a Step Fractional Brownian Motion.", Statistical Inference for Stochastic Processes, Vol. 3, Issue 1/2, p.101-111, 2000.

[10] Benassi, A. and Deguy, S., 1999. "Multi-scale fractional Brownian motion: definition and identification." Preprint LAIC.

[11] Benassi, A., Jaffard, S. and Roux, D., "Elliptic Gaussian random processes", Rev. Mathemàtica Iberoamericana, 13, No 1, p. 19-90, 1997.

[12] Bertrand, P."A local method for estimating change points: the hat-function ", Statistics 34, p.215-235, (2000).

[13] Bertrand, P., Bardet, J.M, Dabonneville, M., Mouzat, A. and Vaslin, P., 2001. Automatic determination of the different control mechanisms in upright position by a wavelet method, I.E.E.E. Engineering in Medicine and Biology Society.

[14] Billingsley, P., 1968. Convergence of Probability Measures. New-York. Wiley.

[15] Cheridito, P., 2003. "Gaussian moving averages, semimartingales and option pricing." Stochastic Processes and their Applications, 109(1), p. 47-68.

[16] Collins J.J. and De Luca C.J., 1993. Open-loop and closed-loop control of posture: A random walk analysis of center-of-pressure trajectories. Experimental Brain Research 9, p. 308-318.

[17] Cramér, H.and Leadbetter, M. R., 1967. Stationary and related stochastic processes. Sample function properties and their applications. Wiley and Sons.

[18] Dahlhaus R., 1989. Efficient parameter estimation for self-similar processes. Ann. Statist. 17, p. $1749-1766$

[19] Delbaen, F. and Schachermayer, W., 1994. "A general version of the fundamental theorem of asset pricing", Math. Annals, (1994) p. 463-520.

[20] Flandrin, P., 1992. Wavelet analysis and synthesis of fractional Brownian motion. IEEE Trans. on Inform. Theory 38, p. 910-917. 
[21] Fox, R. and Taqqu, M.S., 1986. Large-sample properties of parameter estimates for strongly dependent Gaussian time series. Ann. Statist. 14, p. 517-532.

[22] Frisch, U., 1995. Turbulence, the legacy of A.N. Kolmogorov, Cambridge University Press.

[23] Giraitis L. and Surgailis D., 1990. A central limit theorem for quadratic forms in strongly dependent linear variables and its applications to the asymptotic normality of Whittle estimate. Prob. Th. and Rel. Fields. 86, p. 87-104.

[24] Mandelbrot, B. and Van Ness J., 1968. Fractional Brownian motion, fractional noises and applications. SIAM review 10, p.422-437.

[25] Papanicolaou, G. and Sølna, K., 2002. Wavelet Based Estimation of Local Kolmogorov Turbulence, , in Long-range Dependence: Theory and Applications, P. Doukhan, G. Oppenheim and M.S. Taqqu editors, Birkhäuser, p. 473-506

[26] Peltier, R., and Lévy Véhel, J., "Multifractional Brownian motion: definition and preliminary results", Rapport technique INRIA, 1996.

[27] Pipiras, V. and Taqqu M. S., 2004. "Stable stationary processes related to cyclic flows", The Annals of Probability. 23, Nb. 3A, p. 2222-2260.

[28] Rogers, L.C.G., 1997. Arbitrage with fractional Brownian motion. Mathematical Finance 7, p. 95-105.

[29] Samorodnitsky, G. and Taqqu M. S., 1994. Stable non-Gaussian Random Processes, Chapman \& Hall.

[30] Willinger, W., Taqqu,M.S. and Teverovsky, V., 1999. Stock market price and long-range dependence, Finance and Stochastics, 1, p. 1-14 